\title{
Bus energy consumption for multilevel signals
}

\begin{abstract}
A comprehensive analysis of energy consumption for voltage-mode multilevel signals on a nanometer-technology bus is presented. A transition-dependent model is used which allows simplified calculation of the energy consumption. The accuracy of the approach is demonstrated using circuit simulations of three different electrical models of the bus, namely, lumped-C, distributed-RC, and distributed-RLC networks. We also verify that bus energy consumption is independent of driver resistance, as predicted by the model. Finally, we present a comparative analysis of power consumption for multilevel and binary buses.
\end{abstract}

Keyword: Energy consumption; Multilevel signals; Nanometer technology 